\title{
POSITRON ANNIHILATION IN AND ADIABATIC COMPRESSIBILITY OF ETHYLENE GLYCOL AQUEOUS SOLUTIONS
}

\author{
A. Baranowski, K. Jerie \\ Institute of Experimental Physics, University of Wrocław \\ pl. Maxa Borna 9, 50-204 Wrocław, Poland \\ J. GlińsKi AND K. OrZeChowsKi \\ Institute of Chemistry, University of Wrochaw \\ F. Joliot-Curie 14, 50-383 Wrocław, Poland
}

The positron annihilation lifetime and ultrasonic velocity in aqueous ethylene glycol solutions were measured. A comparison between the concentration and temperature dependences of the above parameters suggests that there is a competition of hydrophobic (interstitial) and hydrophylic (substitutional) hydration.

PACS numbers: $61.25 . \mathrm{Em}, 78.70 . \mathrm{Bj}$

\section{Introduction}

The thermodynamic properties of aqueous solutions of molecules having both polar and non-polar components at low concentrations are predominantly determined by hydrophobic interactions, which result in enhancement of water structure in the vicinity of the guest molecule. Such stabilization of water structure gets support by NMR [1] and IR [2] spectroscopy, as well as by X-ray diffraction [3] or molecular dynamics [4]. Many of organic compounds form clathrate hydrates in the solid state, thus attempts to explain the behaviour of liquid solutions in terms of clathrate-like structures seem to be more legitimate than interpretations based on other concepts.

The ultrasonic properties of dilute aqueous solutions of polar organic compounds are well known $[5,6]$. They usually show absorption maxima with increasing solute concentration, whereas their adiabatic compressibility isotherms intersect before reaching minimum values [7]. Both the intersection concentration and the absorption maximum are shifted to lower concentrations of the organic solute with its increasing hydrophobic character; the concentration of maximum 
absorption is somewhat higher than that of the intersection. According to Endo [8] the intersection of the compressibility isotherms should be related to the formation of pseudoclathrate (i.e. clathrate-like) structures. In contrary, Baumgartner and Atkinson [9] relate the formation of such structures to the minima of the compressibility isotherms, while according to Andreae et al. [10] the compressibility minimum can be explained in terms of solute-solvent complex formation.

However, much less investigations were devoted to the aqueous systems, where the solute molecule has no hydrophobic part. In this case, formation of liquid clathrate-like structures is not probable, because the process of building up the solute in liquid water framework is energetically more profitable than breaking the solvent structure and building a new one.

In the present paper, using ethylene glycol as the solute, we have tried to examine this kind of interactions comparing the ultrasonic and positron annihilation data.

\section{Experimental}

\subsection{Materials}

Ethylene glycol (pure for analysis, POCh, Poland) was used without further purification. Water was doubly distilled. Freshly prepared chemicals were used for preparing the samples.

\subsection{Density measurements}

Densities were measured using the vibrating tube densitometer type MG-2 (ECOLAB, Poland) in a temperature range $15^{\circ}$ to $35^{\circ} \mathrm{C}$. The reproducibilities of the densities were much better than $\pm 0.01 \mathrm{~kg} \mathrm{~m}^{-3}$.

\subsection{Sound velocity}

The ultrasonic velocities were measured with an accuracy of not less than $\pm 0.05 \mathrm{~m} \mathrm{~s}^{-1}$ using MT-2 (ECOLAB, Poland) automatic phase velocimeter.

\subsection{Positron annihilation}

The annihilation measurements were carried out at room temperature (approx. $20^{\circ} \mathrm{C}$ ) with a standard measuring device based on the "fast-slow" coincidence technique as described elsewhere [11].

\section{Experimental results}

\subsection{Ultrasonic properties of the system water-ethylene glycol}

The adiabatic compressibility coefficients $\beta$ were calculated from Laplace's equation

$$
\beta=\left(d c^{2}\right)^{-1},
$$






Fig. 1. The adiabatic compressibility coefficient of water-ethylene glycol mixtures vs. concentration. $\Delta-15^{\circ} \mathrm{C}, \mathrm{O}-20^{\circ} \mathrm{C}, \nabla-25^{\circ} \mathrm{C}, \diamond-30^{\circ} \mathrm{C}, \square-35^{\circ} \mathrm{C}$.

where $c$ is the ultrasonic velocity and $d$ is the density of the liquid. The concentration dependence of the compressibility within the low organic solute concentration region, which is of interest for the further discussion, is shown in Fig. 1.

The adiabatic compressibilities plotted against the mole fraction of glycol show two characteristic features: (i) each compressibility isotherm has a minimum shifting from $X_{\text {glycol }} \approx 0.4$ at $308.15 \mathrm{~K}$ to higher concentrations with decreasing temperature, and (ii) the isotherms intersect approximately at a common point (within the temperature range covered by our measurements) corresponding to $X_{\text {glycol }} \approx 0.11$.

\subsection{Positron annihilation parameters}

Two components are expected in an annihilation time spectrum: a short-lived one $\tau_{1}$, with intensity $I_{1}$, connected with the free positron annihilation, and a long-lived component $\tau_{2}$, with intensity $I_{2}$, distinctly separated from the former one and assigned to the pick-off process of the ortho-positronium [11].

The short-lived component is a combination of two processes: free positron annihilation and that of para-positronium. While it is very difficult to distinguish between them, we therefore will consider the short-lived component as one process only. Thus, the time spectra obtained, after making some allowances for "source" contribution [11], were analyzed with regard to the two components $\tau_{1}$ and $\tau_{2}$. The last one, of intensity $I_{2}$, was attributed to ortho-positronium annihilating entirely in the pick-off process [12]. In this treatment the condition $I_{1}+I_{2}=100 \%$ is fulfilled. The results are collected in Figs. $2 \mathrm{a}-\mathrm{c}$. 

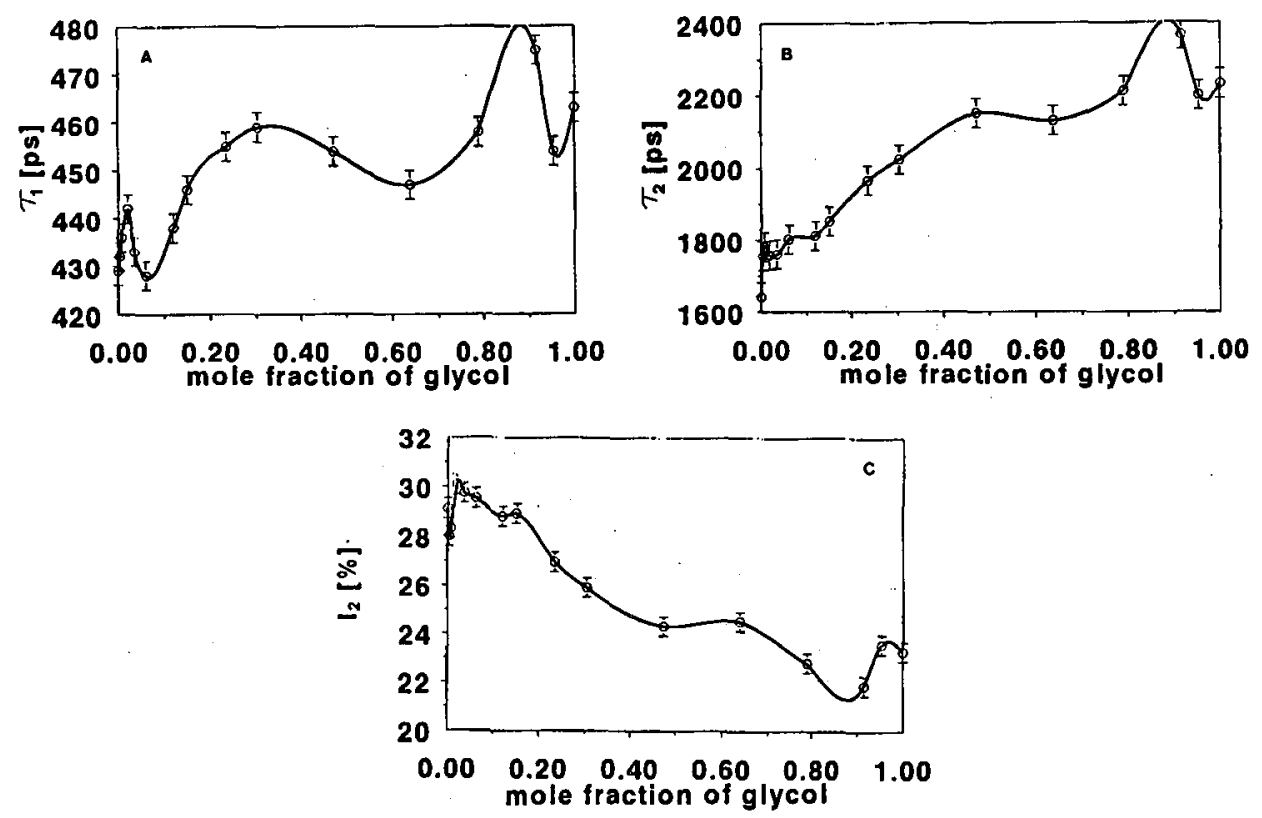

Fig. 2. The results of positron annihilation measurements vs. molar fraction of glycol in water (at room temperature). Lines are drawn arbitrarily (third-order polynomial): (a) lifetimes of the short-lived component of the annihilation spectrum, (b) lifetimes of the long-lived component, (c) intensity of the latter.

The distinct extrema in $\tau_{1}$, maximum in $I_{2}$, and plateaux in $\tau_{2}$, occurring at $X_{\text {glycol }}$ below 0.2 seem to be significant.

\subsection{Density}

The plot of density vs. concentration varies smoothly in the whole concentrations range (Fig. 3). The lack of maxima of these plots may indicate that strong hydrates are not formed in this system.

\section{Discussion}

\subsection{Adiabatic compressibility}

To the best of our knowledge, the formation of clathrate hydrates of ethylene glycol has not been reported in literature yet. This suggests that either glycol does not form inclusion compounds of this type with water or they are too unstable to be isolated in solid state. The former seems more probable, while the molecule of the solute has no hydrophobic part able to be hydrophobically hydrated. 


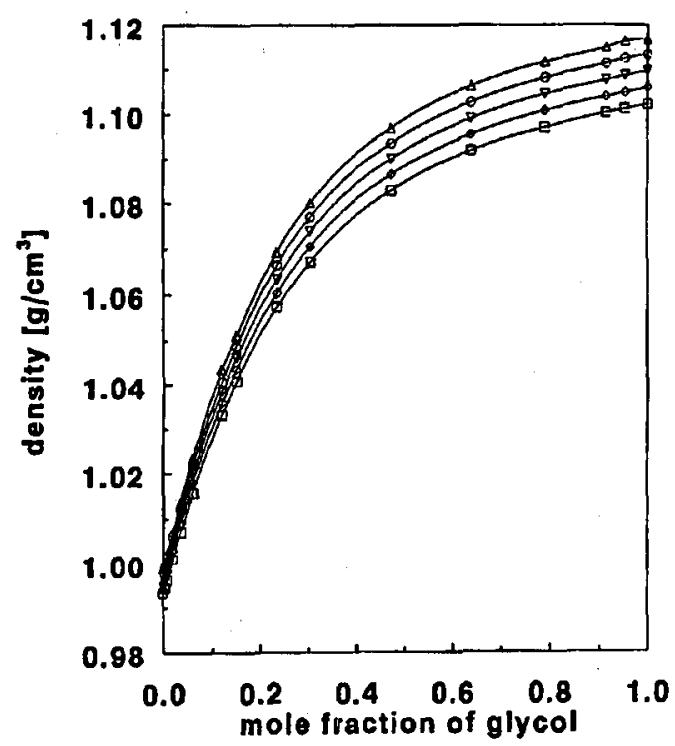

Fig. 3. The densities of ethylene glycol + water mixtures vs. glycol concentration. $\Delta-15^{\circ} \mathrm{C}, \mathrm{O}-20^{\circ} \mathrm{C}, \nabla-25^{\circ} \mathrm{C}, \diamond-30^{\circ} \mathrm{C}, \square-35^{\circ} \mathrm{C}$.

According to the concept of "iceberg formation" of Frank and Evans [13] or to that of hydrophobic hydration formulated by Franks [14], when an organic compound is dissolved in water, the water structure is modified around the non-polar parts of the solute molecule in the direction of greater "crystallinity". Thus, the water structure in the vicinity of the guest organic molecule is determined mainly by the hydrophobic moiety of the guest, the latter being accommodated in the cavities of the open hydrogen-bonded water network, which in pure water are occupied most probably by "monomeric" $\mathrm{H}_{2} \mathrm{O}$ molecules [15]. This physical picture seems to interpret reasonably some properties of aqueous solutions of non-electrolytes, e.g. p-dioxan [16], tetrahydrofuran [11], ethanol [17] and some amines [18].

According to Endo [8], the intersection of adiabatic compressibility isotherms should be attributed to the formation of clathrate-like structures in liquid aqueous system. A similar structural interpretation was suggested in Refs. [17] and [19]. Thus, the intersection point concentration indicates clathrate stoichiometry of 1 glycol: $8 \mathrm{H}_{2} \mathrm{O}$.

Most of water-soluble polar compounds form in solid state cubic lattices of two types: of $\mathrm{CsCl}$ type (structure I) and of diamond type (structure II) [20-23]. The details of these structures and the stoichiometries corresponding to different structural forms of clathrate hydrates can be found in Ref. [11]. The crossing point concentration $X_{\text {glycol }}=0.11$ may be attributed to the structure $\mathrm{I}$ of $\mathrm{CsCl}$ type (lattice dimension $a=1.2 \mathrm{~nm}$ ). The unit cell of this structure consists of 46 lattice water molecules (vertices) that form 2 pentagonal dodecahedra with a free diameter of $0.5 \mathrm{~nm}$, and 6 tetrakaidecahedra which are approximately oblate 
with free diameters of 0.53 and $0.64 \mathrm{~nm}$. Thus, the stoichiometry of clathrate may depend on the size of a solute molecule; small molecules may occupy all the voids, while greater can be accommodated only in the larger ones. In the latter case, smaller voids may be empty and/or occupied by "monomeric" water molecules. The resulting stoichiometries are as follows:

Occupation of the voids of structure I

(i) All voids occupied by the solute

(ii) Larger voids occupied by the solute, smaller voids empty

(iii) Larger voids occupied by the solute, smaller voids occupied by "monomeric" water molecules
Resulting stoichiometry

$X \cdot 5.75 \mathrm{H}_{2} \mathrm{O}$,

$X \cdot 7.65 \mathrm{H}_{2} \mathrm{O}$,

$X \cdot 8 \mathrm{H}_{2} \mathrm{O}$.

The ethylene glycol molecule has no hydrophobic group. Taking into consideration the capability of the hydroxyl group for hydrogen bond interaction with the surrounding water molecules, one has to assume that in the system under investigation the hydrophylic hydration should be dominating. The importance of hydrophylic interactions leading to the replacement of host lattice water molecules by the $-\mathrm{O}-$ and $-\mathrm{NH}-$ groups was stressed by McMullan et al. [24] and by Glew [25].

It should be stressed, too, that a very similar experimental picture of positron annihilation parameters has been obtained for water-methanol system [26]. Moreover, the stoichiometry resulting from the concentration corresponding to the intersection of compressibility isotherms is almost the same (1:8). However, in Ref. [26] this was interpreted in terms of a very small hydrophobic part $\left(-\mathrm{CH}_{3}\right)$ of the solute molecule, while in the case of ethylene glycol there is no distinct hydrophobic part to be hydrated at all.

It is worth noting that the compressibility value at the crossing point ( $3.65 \times$ $10^{-10} \mathrm{~m}^{2} \mathrm{~N}^{-1}$ ) is much lower than that found by von Stackelberg for the solid clathrates of structure I $\left(>4.0 \times 10^{-10} \mathrm{~m}^{2} \mathrm{~N}^{-1}\right)[22,23]$. The lower compressibility means more compact (dense) structure than open-work one of liquid clathrates. In other words: the structure formed at $X_{\text {glycol }} \approx 0.11$ is too strong (in the sense of solute-solvent, as well as solvent-solvent interactions) than those typical of liquid clathrates.

The role of hydrophylic interactions was investigated recently by Jerie et al. [27] in water-formamide system. The lack of hydrophobic hydration yielded the experimental picture resembling a bit that presented in this paper, concentration corresponding to the intersection of compressibility isotherms is very high ( $X_{\text {formamide }} \approx 0.13$ ) and plots of annihilation parameters are different in their shapes than those obtained in the systems, where strong hydrophobic interactions occur.

Concluding, the structure of dilute aqueous solutions of ethylene glycol is untypical. Although the concentration of the intersection of compressibility isotherms could suggest formation of liquid clathrates type I, in fact no such structure can 
be formed, because no hydrophobic hydration is possible. The ultrasonic velocity data give not enough information for proper interpretation and one needs more experimental support.

\section{Acknowledgments}

This work was supported by the State Committee for Scientific Research (Republic of Poland) grant no. PB 1168/2 and GBW/94/5/IFD.

\section{References}

[1] E. von Goldammer, H.G. Hertz, J. Phys. Chem. 74, 3734 (1970).

[2] W.A.P. Luck, D. Schiöberg, Adv. Mol. Relaxational Processes 14, 277 (1979).

[3] M.C.A. Donkersloot, J. Solution Chem. 8, 293 (1979).

[4] A. Geiger, A. Rahman, F.H. Stillinger, J. Chem. Phys. 70, 263 (1979).

[5] K.G. Breitschwerdt, in: Structure of Water and Aqueous Solutions, Ed. W.A.P. Luck, Verlag Chemie GmbH and Physik Verlag GmbH, Weinheim 1974, p. 473.

[6] F. Koller, in: Structure of Water and Aqueous Solutions, Ed. W.A.P. Luck, Verlag Chemie GmbH and Physik Verlag GmbH, Weinheim 1974, p. 491.

[7] K. Jerie, A. Baranowski, B. Rozenfeld, J. Gliński, S. Ernst, Phys. Scr. 35, 729 (1987).

[8] H. Endo, Bull. Chem. Soc. Jpn. 16, 1586 (1973).

[9] E.K. Baumgartner, G.J. Atkinson, J. Phys. Chem. 75, 2336 (1971).

[10] J.H. Andreae, P.D. Edmonds, J.F. McKellar, Acustica 15, 74 (1965).

[11] K. Jerie, A. Baranowski, B. Rozenfeld, S. Ernst, J. Gliński, Acta Phys. Pol. A 64, 77 (1983).

[12] B. Levay, A. Vertes, P. IIautojärvi, J. Phys. Chem. 77, 2229 (1973); B. Levay, P. Hautojärvi, Radiochem. Radioanal. Lett. 10, 309 (1972); R.E. Wild, H.J. Ache, Radiochem. Radioanal. Lett. 23, 249 (1975).

[13] H.S. Frank, M.W. Evans, J. Phys. Chem. 13, 507 (1945).

[14] F. Franks, Ann. N.Y. Acad. Sci. 125, 277 (1965).

[15] H.S. Frank, A.S. Quist, J. Chem. Phys. 34, 604 (1961); V.N. Yashkistshev, O.Ya. Samoilov, Zh. Strukt. K'him. 4, 502 (1963).

[16] S. Ernst, J. Gliński, Chem. Tech. 29, 51 (1977).

[17] K. Jerie, A. Baranowski, S. Ernst, J. Gliński, Acta Phys. Pol. A 69, 81 (1986).

[18] S. Ernst, J. Glinski, Polish J. Chem. 56, 339 (1982).

[19] S. Ernst, J. Gliński, Mater. Sci. 3, 68 (1977).

[20] G.A. Jeffrey, R.K. McMullan, Prog. Inorg. Chem. 8, 43 (1967).

[21] S.Sh. Byk, Ju.F. Makogon, V.I. Fomina, Gazovye Gidraty, Izd. Khimia, Moscow 1962, pp. 12-16.

[22] H. Müller, M. von Stackelberg, Naturwissenschaften 39, 20 (1952). 
[23] M. von Stackelberg, H. Müller, Z. Elektrochem. 58, 25 (1954).

[24] R.K. McMullan, T.H. Jordan, G.A. Jeffrey, J. Chem. Phys. 47, 1218 (1967).

[25] D.N. Glew, Nature 184, 545 (1959).

[26] K. Jerie, A. Baranowski, B. Rozenfeld, S. Ernst, B. Jeżowska-Trzebiatowska, J. Gliński, Acta Phys. Pol. A 66, 167 (1984).

[27] K. Jerie, A. Baranowski, J. Gliński, K. Orzechowski, J. Phys. C IV-3, 135 (1993). 